Reprod. Nutr. Dévelop., 1980, 20 (3 B), 871-880.

\title{
Le développement des jeunes enfants élevés en enceinte étanche stérile : problèmes psychologiques ef relation parentale
}

par Danielle RAPOPORT, Françoise WEIL-HALPERN, C. GRISCELLI

Groupe de Recherches d'Immunologie

et de Rhumatologie Pédiatriques, INSERM U 132,

Hôpital Necker Enfants Malades,

149 rue de Sèvres, 75730 Poris Cedex 15.

Summary. The development of young children maintained in a Trexler's isolator: Psychological problems and parental relationship.

Five children with severe immunodeficiency who received a transplantation of lymphoid cells were placed in a Trexler's isolator for several months during the time necessary to obtain an immunological reconstitution. We have observed these patients longitudinally (from 2 to 7 years), and have considered the various parameters necessary to preserve a satisfactory psychological development and a good adaptative capacity. Among these, the careful and affective attention of nurses and parental behaviour appeared to be the most important. Excellent development was obtained in three patients who had no severe organic difficulties and for whom the above parameters were positive. The cause of two unsatisfactory results seemed to be due to intricate multifactorial events, including parental deprivation.

\section{Introduction}

Les enfants atteints de déficits immunitaires congénitaux profonds peuvent bénéficier d'une transplantation médullaire, sans laquelle ils décèdent très rapidement d'infections. La durée nécessaire à la prise de greffe, pour aboutir à une reconstitution satisfaisante des moyens de défense, est cependant longue. Afin de réduire les risques d'infections, l'enfant doit séjourner plusieurs mois de la première année de vie en enceinte étanche de plastique transparent (d'où le nom de «bulle» couramment utilisé pour désigner les enceintes axéniques). On impose ainsi au nourrisson de vivre dans un espace restreint et confiné, qui limite considérablement les stimulations corporelles, affectives et sociales dont il a besoin.

Face à ces risques de carences, dont on peut craindre les conséquences et dont on sait reconnaître les manifestations (Bowlby, 1978) nous avons tenté d'associer à l'extrême surveillance médicale qui entoure ces enfants une aussi grande vigilance psychologique. Nous rapportons ici cinq observations ef l'expérience acquise durant ces sept dernières années. 


\section{Matériel et méthodes}

Dans la population de 25 enfants qui, de 1972 à 1975, ont effectué un séjour en enceinte étanche stérile, nous avons pu faire l'éłude psychologique longiłudinale de 5 d'entre eux, sélectionnés sur des critères communs. Tous ont été traités, ont séjourné en enceinte étanche stérile plusieurs mois de la première année de vie, et ont pu être suivis après la levée de l'isolement en raison d'une bonne évolution organique.

L'enceinte étanche stérile (Intensel Elster, Paris) est une sorte de grande couveuse aux parois de plastique souple. Les relations qui s'établissent entre l'enfant et le monde extérieur se font par le contact au travers des gants, par la vision ef par les sons. Ces derniers ne sont pas déformés par la barrière de plastique, mais ils sont légèrement amplifiés lorsqu'ils sont produits par un contact direct avec la paroi (par exemple lorsqu'on embrasse l'enfant à travers elle). Il existe aussi un bruit de fond permanent produit par les ventilateurs. Les gants ont une importance capitale car ils permettent les contacts corporels. C'est grâce à eux que l'on peut porter l'enfant, le manipuler, le caresser...

Le lieu, où se sont déroulées ces observations se situe dans l'Unité d'Immunologie d'un hôpital pédiatrique. Cette Unité est hautement spécialisée mais elle bénéficie d'aménagements favorables à un climat ouvert et chaleureux. Les locaux sont gais, les familles $y$ viennent sans restriction d'horaires ou de personnes. Les aires de jeux et d'activités pédagogiques ont une place importance dans la vie hospitalière. Les infirmières, les médecins, les psychologues, l'assistante sociale forment une équipe particulièrement homogène et stable. C'est autant de conditions qui facilitent des observations psychologiques longitudinales rendues difficiles par la gravité des maladies traitées. L'évolution des enfants a été suivie en majorité dans le cadre des consultations, et parfois au domicile et à l'école.

Les méthodes d'observation utilisées sont celles de la psychologie clinique (FavezBoutonnier, 1968). Nous avons essayé, pour chaque enfant et dans la mesure du possible, de considérer tous les facteurs qui pouvaient entrer en jeu dans sa situation donnée et dans son évolution, dans une perspective globale, sans privilégier une donnée par rapport à une autre. Nous avons particulièrement considéré : 1) Les caractéristiques médicales propres à chaque cas (type de déficit immunitaire, évolution organique et diversité des traitements proposés). 2) La variété des conditions de séjour en enceinte étanche stérile et la variété des contributions psychologiques et éducatives de l'équipe de soins. 3) Le contexte affectif et socio-familial environnant l'enfant durant son séjour à l'hôpital et à sa sortie. 4) L'état psychologique ef le développement psychomoteur de chaque enfant, évalués à la fois par les méthodes d'observation directe et par les échelles de développement psychométriques d'O. Brunet et d'l. Lézine, adaptation française des baby-tests d'A. Gesell (Lézine, 1974) et appréciés tous les deux mois la première année puis deux fois par an.

Nous devons souligner que les psychologues, très intégrées à l'équipe de soins, ont pu effectuer leurs observations en tenant compte de l'ensemble des facteurs médicaux, institutionnels, familiaux et individuels. De ce fait, les psychologues, constamment informées des données purement médicales, ont été directement impliquées tout au long de ces observations. La confrontation permanente avec l'ensemble de l'équipe a été un garant d'objectivité. 


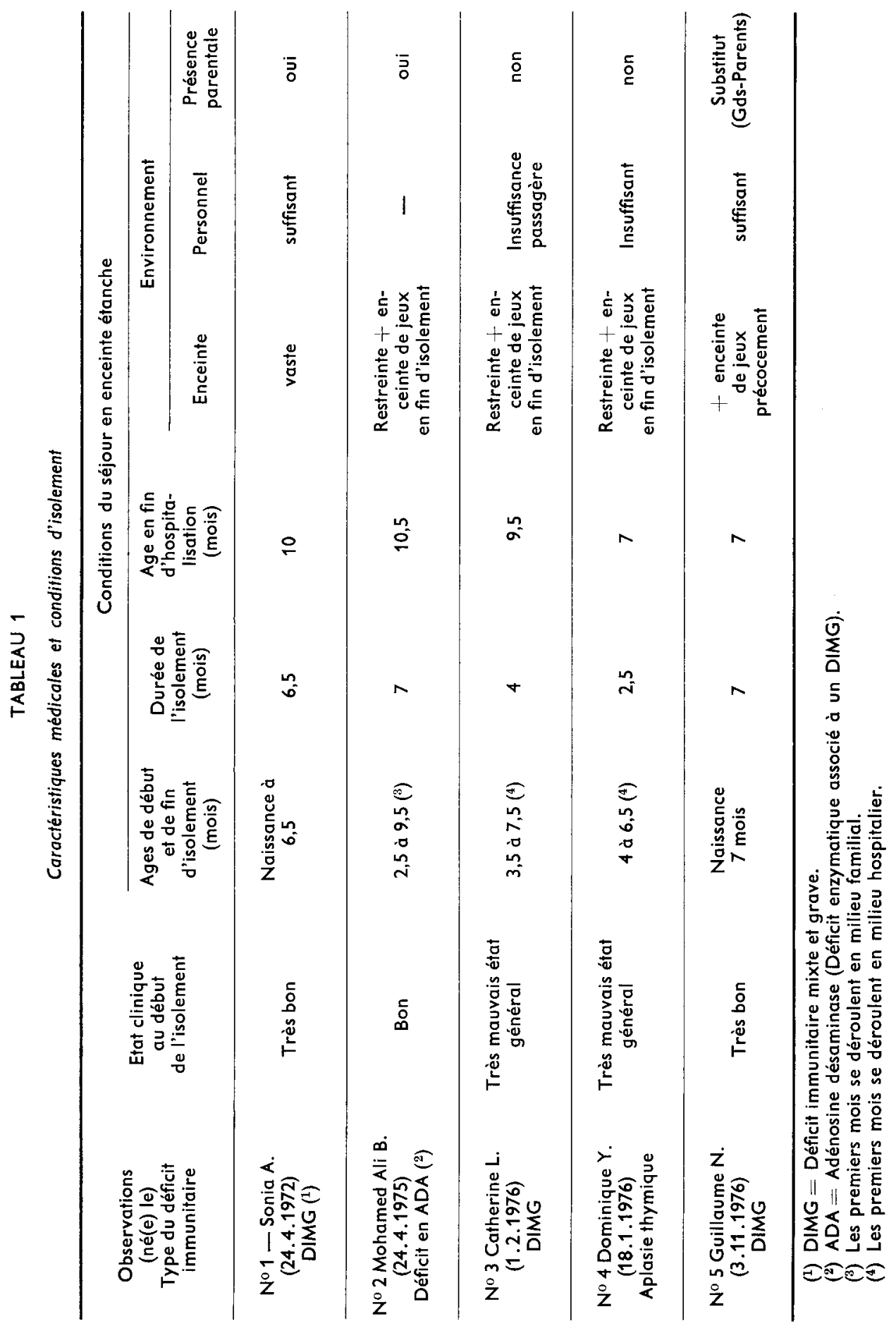




\section{Observations et résultats.}

Les principales données sont résumées dans les tableaux 1 et 2 .

TABLEAU 2

Evolution psychologique après l'hospitalisation

\begin{tabular}{|c|c|c|c|}
\hline $\begin{array}{l}\text { Observations } \\
\text { (Né(e) le) }\end{array}$ & $\begin{array}{l}\text { Caractéristiques } \\
\text { socio-affectives } \\
\text { de la famille }\end{array}$ & $\begin{array}{l}\text { Développement } \\
\text { psychologique } \\
\text { et adaptation } \\
\text { (zones de QD et QI) }\end{array}$ & Scolarité actuelle \\
\hline $\begin{array}{l}\text { No } 1 \text { Sonia A. } \\
(24.4 .1972)\end{array}$ & Très bonnes & $\begin{array}{l}\text { Excellent } \\
(105-115)\end{array}$ & $C E_{1}$ à 7 ans \\
\hline $\begin{array}{c}\text { No } 2 \text { Mohamed Ali B. } \\
(25.4 .1975)\end{array}$ & Très bonnes & $\begin{array}{c}\text { Bon } \\
(100-105)\end{array}$ & $\begin{array}{l}2^{e} \text { section de jardin d'en- } \\
\text { fants }\end{array}$ \\
\hline $\begin{array}{c}\text { No } 3 \text { Catherine L. } \\
(1.2 .1976)\end{array}$ & $\begin{array}{l}\text { Hospitalisée } \\
\text { en pouponnière }\end{array}$ & $\begin{array}{c}\text { Moyen } \\
\text { Troubles du comportement } \\
(75-85)\end{array}$ & $\begin{array}{l}\text { Non scolarisée à } 3 \text { ans ef } \\
\text { demi (parents nomades) }\end{array}$ \\
\hline $\begin{array}{c}\text { No } 4 \text { Dominique } Y . \\
18.1 .1976)\end{array}$ & $\begin{array}{l}\text { Hospitalisé } \\
\text { en pouponnière }\end{array}$ & $\begin{array}{c}\text { Mauvais } \\
\text { Evolution psychotique } \\
(75-85 \text { puis } 50)\end{array}$ & $\begin{array}{l}\text { Non scolarisé, suivi en éfa- } \\
\text { blissement spécialisé par } \\
\text { le secteur d'hygiène men- } \\
\text { tale }\end{array}$ \\
\hline $\begin{array}{c}\text { No } 5 \text { Guillaume N. } \\
(3.11 .1976)\end{array}$ & Très bonnes & $\begin{array}{l}\text { Très bon } \\
(100-110)\end{array}$ & $\begin{array}{l}\text { Entre au jardin d'enfants à } \\
3 \text { ans }\end{array}$ \\
\hline
\end{tabular}

Observation no 1 : Sonia $A$, née le 24.4.1972, a été placée en couveuse dès sa naissance ef en enceinte étanche stérile à 25 jours. Elle est le cinquième enfant d'une fratrie où seul le frère aîné est vivant. Trois enfants sont décédés du même déficit immunitaire que Sonia. Une compatibilité parfaite entre le frère aîné et Sonia permet d'envisager une transplantation médullaire. Les parents, d'origine tunisienne, travaillent en France et sont de niveau socio-économique modeste. Ils seront toujours confiants, chaleureux et présents. Sonia est le premier cas traité par notre équipe (et le troisième cas mondial) et elle sera particulièrement investie et stimulée. De plus, elle bénéficie d'un prototype d'enceinte étanche stérile particulièrement spacieux et bien aménagé. Très vite, son développement psychomoteur, son éveil et sa sociabilité s'avèrent très précoces et son rythme de progression excellent, comme le traduisent les quotients de développement effectués tous les deux mois dans la zone constante de 105-115. Elle ne rencontre pas de difficultés organiques importantes et sort de l'enceinte à l'âge de 6 mois et demi. Elle va rester cependant dans le service durant trois mois et demi car la surveillance médicale de ce cas sans précédent est encore au premier plan des préoccupations. Paradoxalement, c'esł durant cette période que Sonia progresse le moins vite, devient moins active et qu'apparaissent de petites stéréotypies traduisant des risques de premières carences socio-affectives. C'est qu'elle est alors l'objet de moins de stimulations, de moins d'attention que durant son séjour en enceinte. Le retour au foyer familial, à 
l'áge de 10 mois, est d'emblée bénéfique et la vie de cette superbe petite fille se normalise très rapidement. Sonia est âgée actuellement de 7 ans et scolarisée sans difficulté. Elle a eu une petite søur non atteinte. La surveillance médicale est réduite à deux consultations par an.

Observation no 2 : Mohamed-Ali B., né le 25.04.75, au Maroc, est le sixième enfant d'une fratrie où seul le frère aîné est vivant et présente les mêmes antigènes d'histocompatibilité permettant de réaliser une transplantation médullaire. Quatre enfants sont décédés du même déficit immunitaire dont Mohamed Ali est atteint. Ses parents acceptent, malgré l'éloignement, de placer Mohamed Ali en enceinte étanche stérile à l'âge de 2 mois et demi. Le niveau socio-économique de la famille est aisé et permet aux parents de venir alternativement le voir, lors de courts séjours. Ils sont l'occasion d'une relation affective intense et stimulante, et l'on observe que l'enfant souffre des séparations. Son développement somatique n'est pas parfait en raison de difficultés alimentaires et digestives. II est confiné dans une enceinte de petit volume et ce n'est que trois semaines avant la sortie qu'une seconde enceinte « aire de jeux » est adjointe à la première. Il s'épanouit beaucoup en découvrant un nouvel espace qui préfigure le monde extérieur qu'il retrouve à l'âge de neuf mois et demi. II reste, par mesure de précaution, encore un mois dans le service, en compagnie de sa mère et il retrouve son frère à Casablanca à l'âge de 10 mois et demi. Chez cet enfant hypersensible et très émotif, dont le développement psychomoteur et l'éveil ont toujours été satisfaisants avec des quotients de développement entre 100 et 105, la normalisation de la vie quotidienne se fait lentement. Mais l'absence de complications organiques ultérieures, une relation parentale stable, sécurisante, sans hyperprotection, ont permis à Mohamed de sortir peu à peu de sa dépendance, d'aller en jardin d'enfants à temps complet et d'avoir l'autonomie de ses quatre ans.

Observation no 3 : Catherine L., née le 1.02 .76 est la huitième enfant d'une fratrie dont trois enfants, nés d'une première union, sont bien portants. D'une seconde union, deux sont décédés. Une des sœurs non atteintes peut être utilisée comme donneur de moelle du fait d'une compatibilité tissulaire. Catherine a un premier développement difficile. Les trois premiers mois de la vie se déroulent dans un service de pédiatrie en province. Sa famille vit dans un camp de nomades en déplacement constant et a peu de contacts avec l'enfant durant cette période. En raison du diagnostic de déficit immunitaire réalisé dans le service du Professeur Jean (Montpellier), elle nous est adressée pour être placée en enceinte étanche stérile à l'âge de cinq mois 21 jours. C'est seulement à la fin du cinquième mois que son état général s'améliore enfin. Durant deux mois, Catherine profite peu, sur le plan psychomoteur, de son amélioration organique. Elle ne peut bénéficier de la seule aire de jeux que possède alors l'Unité et qui est utilisée par un autre enfant. Le changement des infirmières est plus important en raison de la période des vacances (été 1976). La famille ne peut toujours pas venir la voir. Aussi reste-t-elle assez passive, hypotonique, avec de petites stéréotypies des mains et du corps dès que l'on ne l'occupe pas. Sinon, son contact socio-affectif est excellent mais elle attire peu l'attention sur elle et a peu d'initiative. Le retour de deux infirmières établissant avec elle une relation stable et privilégiée, l'adjonction de l'enceinte de jeux permettent d'obtenir une transformation rapide de l'enfant. $A 7$ mois 23 jours, à la sortie de l'enceinte, le quotient de développement psychomoteur de Catherine est de 
85 alors qu'il était inférieur à 50 lors de son arrivée. Elle quitte notre service à l'âge de 9 mois et demi, pour une pouponnière en raison des difficultés familiales, aggravées par le décès du père. Catherine, alors douce, très sociable, est cependant vite angoissée par les inconnus, à un âge où tout changement risque d'être traumatisant. Six mois plus tard, lorsqu'elle esł réhospitalisée, elle manifeste des réactions d'une extrême violence. On peut considérer comme positives les réactions de l'enfant qui refuse les situations de changement ef les retours en milieu hospitalier, mais son instabilité, son exigence, voire son agressivité pour obtenir à tout prix une relation individualisée ne sont pas sans rappeler les troubles observés chez les enfants élevés exclusivement en institution (David et Appell, 1962, 1973). Actuellement, Catherine est de retour dans sa famille, mais on ignore encore si celle-ci peut offrir à l'enfant la relation thérapeutique ef restructurante dont elle a besoin.

Observation No 4 : Dominique $Y$., né le 18.01.76, a une sœur aînée bien portante âgée de 2 ans et 10 mois. L'enfant présente des difficultés organiques graves dès le $4^{e}$ jour de la vie : des convulsions hypocalcémiques, un reflux pharyngonasal avec insuffisance vélopalatine ef une anomalie vasculaire (artère sous-clavière aberrante). L'absence de thymus, radiologiquement détectée, évoque un syndrome de déficit immunitaire rapidement confirmé par les examens biologiques. La gravité de son état nécessite un maintien en milieu hospitalier jusqu'à l'âge de quatre mois (service du Professeur Jean, Montpellier) d'où la décision de nous l'adresser est prise pour le placer en enceinte étanche stérile. Une alimentation parentérale continue est instaurée en raison des troubles digestifs majeurs que présente le malade. Le traitement par injections de facteurs thymiques permet, conjointement aux traitements symptomatiques, d'obtenir une rapide amélioration de l'état clinique et immunologique et malgré la lourdeur des traitements comme de la haute technicité qu'ils impliquent, les infirmières investissent-elles de plus en plus Dominique, encouragées par son évolution rapidement favorable. A l'âge de six mois et demi, à la sortie de l'enceinte, il a un éveil psychomoteur voisin de la normale en ce qui concerne son intelligence sensori-motrice (QD partiel dans la zone 85-90); son contrôle posturo-moteur est beaucoup plus fragile, comme le traduit le QD partiel égal à 70 : Dominique est actif, joueur et bavard en position couchée mais il reste passif et mal à l'aise en position assise. Les parents, domiciliés en province, n'ont pas pu prendre le relais. On découvre que leurs possibilités intellectuelles sont limitées et qu'ils comprennent mal les difficultés de leur enfant. De plus, le développement intellectuel de leur fille ne semble pas parfaitement normal. Dominique esł confié à une pouponnière, car ni sa grand-mère, qui lui porte pourtant un grand intérêt, ni ses parents ne sont en mesure de poursuivre le régime et la surveillance médicale. Revu à l'âge de seize mois, Dominique est un bel enfant, lourd et un peu bouffi, triste. Au cours des mois qui vont suivre, sa passivité va s'aggraver et lorsque nous le revoyons l'année suivante, c'est un enfant psychotique, par moment autistique, qui est actuellement pris en charge par les services d'hygiène mentale de son secteur.

Observation no 5 : Guillaurne N., né le 3.11 .76 (par césarienne axénique pour des raisons purement obstétricales), est aussitôt placé en enceinte étanche stérile. II a un frère aîné bien portant, mais deux enfants sont décédés avant lui de la même affection. Ce seul frère ne peut être retenu comme donneur de moelle, du fait d'une incompatibilité des antigènes tissulaires. Une enquête familiale est menée à la recher- 
che d'un donneur compatible. Cette enquête permet de découvrir qu'une tante maternelle de l'enfant peut être considérée comme donneur idéal. Les parents, redoutant d'avoir à revivre les chocs occasionnés par les décès précédents (dont un est survenu dans cette même Unifé), préfèrent repartir en province. Les grands-parents et la tante se substifuent discrètement. Les parents établissent cependant des contacts téléphoniques fréquents et ne s'excluent en réalité que physiquement. Guillaume restera durant le premier mois dans une sorte de nébuleuse, comme en vie intra-utérine, dormant beaucoup. Assez soudainement, il sort de cet étał à l'âge de un mois et son éveil psychomoteur va se poursuivre normalement. Bénéficiant tôt de l'enceinte de jeux, il devient un enfant actif, joueur et gai mais aussi calme, tranquille, très sécurisé. A l'âge de sept mois, le jour de la sortie, des risques de contagion (varicelle) justifient de le rendre directement à sa famille. Les parents, particulièrement informés de la nécessité de respecter une certaine continuité entre le mode de vie de l'enfant en enceinte étanche stérile et le monde extérieur sauronf l'aider pendant la période de transition. Guillaume va poursuivre, chez lui, avec le même rythme et la même tranquillité, un développement psychomoteur harmonieux, que les consultations bi-annuelles mettent en évidence avec la même constante (QD zone 100-110).

\section{Discussion.}

Notre propos est de considérer la part de la relation parentale dans le développement psychologique ef l'adaptation de cinq enfants soumis à un isolement en enceinte étanche stérile pendant une durée de 2 mois et demi à 7 mois. Si la relation parentale représente l'un des facteurs les plus importants intervenant dans l'évolution psychologique de ces malades, elle doit cependant être considérée dans un ensemble de données techniques, médicales et institutionnelles (Rapoport ef al.).

L'analyse globale de nos résultats fait apparaître que trois des cinq malades, étudiés longitudinalement, ont eu un développement psychologique et une adaptation parfaite. Il semble ainsi que le confinement en enceinte étanche n'est pas, en soi, un facteur qui puisse avoir des conséquences néfastes. Il a pu même être constaté que les meilleurs résultats ont été obtenus chez les trois enfants qui ont été isolés pendant la plus longue durée. Chez les deux autres enfants placés en enceinte étanche alors que leur étał clinique était très médiocre, nous avons pu observer une rapide progression du développement psychomoteur, conjointement à l'amélioration somatique. Une analyse plus fine fait cependant découvrir qu'au-delà d'un âge de développement psychomoteur de 6 mois, peuvent survenir les premiers symptômes de carences marqués par des mouvements stéréotypés passagers, des demandes à autrui de plus en plus fréquemment exprimées. Une certaine insatisfaction apparaît malgré les réponses que les enfants obtiennent.

Les caractéristiques médicales, bien différentes d'un malade à l'autre, représentent un facteur déterminant. En effet, les malades étudiés regroupent trois catégories bien distinctes. Le déficit en ADA ( ${ }^{1}$ ) (Meuwissen et al., 1975), contrairement au DIMG $\left({ }^{2}\right)$

(1) $A D A=$ adénosine désaminase.

(2) DIMG = déficit immunitaire mixte ef grave. 
(Griscelli et al., 1978), est une affection enzymałique qui a d'autres conséquences que celles concernant le système immunologique et, dans l'aplasie thymique ou syndrome de Di George, un retard mental aggravé par les convulsions hypocalcémiques néonatales est décrit (Conley et al., 1979). Ainsi peut-on observer que le seul malade de notre série atteint d'aplasie thymique a effectivement le développement psychologique le plus médiocre. Il est cependant difficile d'imputer aux seules anomalies organiques l'évolution psychotique que nous observons.

Les données institutionnelles nous ont paru tout aussi essentielles. Elles reposent sur la qualité du maternage (Robertson, 1972) réalisé par les infirmières et constituent une sorte de substitut de la relation parentale. Nous avons constamment observé un investissement professionnel et affectif remarquable facilité, il est vrai, dans ces situations encore nouvelles, par l'intérêt thérapeutique et scientifique et par l'enjeu d'une telle entreprise. Cette attitude des infirmières auprès du malade a été primordiale en l'absence des parents ef a parfois persisté lorsque l'investissement de l'infirmière a été tel qu'il lui était difficile de le rompre en leur présence. C'est, en effet, par une vigilance psychologique de tout instant, par une sensibilité aux demandes de l'enfant et par une capacité de compenser le confinement en enceinte que l'infirmière a pu prévenir les risques de carence et de régression. II n'est pas étonnant, du fait de l'implication des infirmières dans les moindres gestes de la vie quotidienne de l'enfant et de la collaboration réciproque avec les psychologues, qu'un esprif constant de recherche ait pu aboutir à de multiples aménagements visant à compenser les risques de confinement. Nous avons pu observer chez deux malades (observations nos 1 ef 3 ), lors d'une diminution momentanée de l'attention des infirmières, une stagnation dans le développement de ces enfants.

La présence des parents auprès de leur enfant durant toute la période de confinement nous a semblé être un élément garant d'une continuité affective indispensable au sortir de l'hospitalisation. Il est important de préciser que la technique d'isolement en enceinte étanche permet aux parents d'être constamment présents sans aucune précaution bactériologique, normalement vêtus, sans les risques de contamination dont on connaît dans d'autres situations le retentissement anxiogène. Ceci permet une précoce et constante participation des parents à la vie de l'enfant en enceinte. Il est bien évident que réciproquement, l'enfant peut ainsi établir précocément les relations naturelles avec ses parents. Les informations d'ordre médical ef technique qui leur sont données préalablement ont l'avantage de réduire les risques de réactions phobiques ef de favoriser une participation active. La levée de l'isolement en présence des parents reste un moment d'intense émotion qui précède la période de transition au cours de laquelle la présence manifeste de l'équipe soignante se fait de plus en plus discrète.

L'étude longitudinale des enfants montre que les capacités d'accueil des parents, lors du refour au foyer fomilial, sont le principal garant d'un développement ultérieur satisfaisant. C'est la qualité des interrelations parents-enfant qui est la source d'une sécurité ef d'une continuité dont l'enfant a besoin dans sa propre histoire. Nous constatons les différences importantes dans la structuration de la personnalité de l'enfant selon la qualité de la relation parentale dont ils ont pu bénéficier. Ainsi, les trois enfants qui ont trouvé un accueil de qualité auprès de leurs parents ont eu un remarquable développement psychologique et une excellente adaptation. De plus, nous 
avons été attentifs au fait que les événements exceptionnels des premiers mois de la vie soient intégrés par l'enfant surtouł grâce au récit que les parents lui en font, s'aidant de photos prises pendant l'isolement en enceinte. Les visites à l'hôpital sont également le prétexte d'une démystification de l'enceinte qu'il retrouve de l'extérieur ef maîtrise. Inversement, les deux enfants qui ont dû être hospitalisés en pouponnière et qui de ce fait n'ont pas bénéficié d'une relation parentale privilégiée, ont eu un développement psychologique moins satisfaisant. L'un d'entre eux, a une évolution psychotique qui pourrait, il est vrai, être en partie expliquée par des anomalies organiques. Ces deux exemples précis tendent à montrer combien de bonnes relations parentales sont essentielles à ce stade.

L'ensemble de notre étude encore limitée à la fois par le nombre de cas étudiés et par la durée de l'étude longitudinale nous permet de conclure préliminairement sur certains points : le confinement en enceinte étanche ne paraît pas, en soi, un élément néfaste à un développement et un comportement adaptatif harmonieux si de nombreuses précautions sont conjointement prises. L'investissement relationnel des infirmières ef la présence parentale nous paraissent jouer ici un rôle de premier plan, touł comme la limitation du confinement qui ne doit pas se prolonger au-delà d'un certain âge de développement (Williamson et al. .1977). Encore faut-il que préside un climat institutionnel qui soustende, pour chacun, une activité intégrée à une cause commune : au-delà de l'obtention d'une reconstitution immunologique, éviter tout facteur qui puisse être la cause d'un développement insuffisant et de troubles de la personnalité. II reste que le meilleur garant d'un bon développement réside en la continuité d'une relation parentale établie précocément et poursuivie harmonieusement.

Colloque "La relation parentale» Nouzilly, France, juin 1979.

Remerciements. - Ce travail a été réalisé avec l'aide de l'INSERM (ATP, 66.78.98) (Unité d'Immunologie et d'Hématologie pédiatriques, Département de Pédiatrie. Hôpital des Enfants Malades, Paris).

\section{Références}

BOWLBY J., 1978. L'aftachement, T. 1. La séparation, T. 2. PUF, Paris.

CONLEY M. E., BECKWITH J. B., MANCER J. F. K., TENCKHOFF L., 1979. The spectrum of the Di George syndrome. J. Pediatr., 94, 883-890.

DAVID M., APPELL G., 1962. Etude des facteurs de carence affective dans une pouponnière, Psychiatr. Enfant, Paris, 4, 407-442.

DAVID M., APPELL G., 1973. Loczy ou le maternage insolife, Ed. du Scarabé, Paris.

FAVEZ-BOUTONNIER J., 1968. La psychologie clinique, Bull. Psychol., Paris, no spéc. $270, \times X \mathbf{1}$ 15-19.

GRISCELLI C., DURANDY A., VIRELIZIER J. L., BALLET J. J., DAGUILLARD F., 1978. Selective defect of precursor $T$ cells associated with apparently normal $B$ lymphocytes in severe combined immunodeficiency disease. J. Pediatr., 93, 404-411. 
LÉZINE I., 1974. Propos sur le jeune enfant, chap. II : Les échelles de développement psychomoteur, Name édit., Paris.

MEUWISSEN H. J., POLLARA B., PICKERING R. J., 1975. Combined immunodeficiency disease associated with adenosine deaminase deficiency (Rep. Workshop held in Albany, New York Oct. 1, 1972) J. Paediat., 86, 169-181.

RAPOPORT D., WEIL-HALPERN F., GRISCELLI C. Effets des restrictions et des carences sur le développement de jeunes enfants atteints de déficit immunitaire devant être élevés en enceinte étanche stérile (en préparation).

ROBERTSON J., 1972. Jeunes enfants à l'hôpital. Ed. du Centurion, Paris.

WILLIAMSON A., MONTGOMMERY J. R., SOUTH M. A., WILSON R., 1977. A special report : Four-year study of a boy with combined immune deficiency maintained in strict reverse isolation from birth. Ped. Res., 11, 63-64. 